\title{
Noise analysis and research on AMT gear shifting
}

\author{
Zhou Jianke \\ Automotive Engineering institute, Jiangxi University of Technology, Nanchang 330098, China
}

Keywords: Noise between shifting; Neural networks; Minicar with automatic shifting; Noise analysis

\begin{abstract}
With the continuous development of vehicle automatic transmission technology and people's pursuing for dynamic and comfort, the quality of gear shifting of automatic transmission vehicles is attached importance to increasingly. The ideal shifting must be smooth, quiet, coherent, natural, etc. This article analyzes the procedure of AMT gear shifting and studies the mechanism of noise generation and fluctuation between the shifting. Here numbers of factors affecting the fluctuation are put forward and the neural network model for noise analysis between the shifting is set up based on plentiful experimental data. It takes AMT vehicles' first gear shifting to second gear as an example to research the effect of each duty parameter on the noise fluctuation between the shifting.
\end{abstract}

\section{Introduction}

Traditional shifting quality refers to how swiftly and firmly gears can be changed without affecting the vehicle's dynamic and its power transmission system [1]. As modern automobile industry develops and vehicle's whole performance is rapidly improved, people expect more about shifting quality whose contents get richer and richer. The solution to improve the shifting quality simply from the angle of ride comfort can't adapt to the demand of all-round improvement of its average level. Modern shifting quality indicates the comfort index including the shifting impact resulting from transmission ratio change and the shifting noise generated by speed fluctuation and vibration, the shifting fuel efficiency and the emission index to consider energy sources and environmental protection, the service life index of the components in power transmission system affected by shifting peak load, and the intelligence and manipulability index to test the responding speed and the satisfaction with the drivers between the shifting. The purpose of shifting quality research is to properly define its evaluation index, to scientifically analyze the relationship and effects among the indicators in different automatic speed control system, and to construct the dynamic comprehensive assessment system of shifting quality so as to lay a theoretic foundation for evaluating objectively the match and calibration technology of power transmission system and the performance of products.

Shifting quality is the key indicator to judge the comprehensive performance of transmissions, which is also the significant basis on which to design, test, match and calibrate, produce and inspect transmissions. As for the profound research on the reasonability of the assessment index and the scientificity of assessment methods, it is crucial to construct an assessment system for shifting quality. A sophisticated overall assessment system lays a theoretical basis for shifting control and guarantees the improvement of the quality.

The specific research on vehicles' shifting noise doesn't spread widely at home or abroad. The study in our country tends to subjective assessment. Since the shifting noise produced by automatic transmission not only involves numerous factors, but it is also related to the subjective feeling of 
both drivers and passengers, when evaluating the shifting noise, domestic researchers mainly adopt the degree of driver's satisfaction as the subjective assessment method, namely, invite some experienced or trained driving evaluators to do real-time assessment and to judge a system shifting noise by grading the test driving.

The overseas shifting noise researches just begin as well, most of which are based on the sound quality itself with the theoretical foundation of Zwicker's sound psychology. The more representative one is the noise research of gearbox ---- double parameters noise assessment method, put forward by Steven Becker and Scott X. Yu. They emphasize human bodies' reaction to noise, especially the wide-band noise, narrow-band noise and low-pass noise. Those three indicators are composed as an acceptable noise quantification index, which can be utilized to replace subjective assessment entirely or partially.

\section{Reasons for generating shifting noise}

AMT is the automatic shifting based on the original manual transmission and dry clutch. The shifting schedule depends on the shifting moment when the vehicle is being driven. When the gear is changed, the controller sends the instruction first, and then the actuators react according to the programmed time and order, and finally the shifting is completed. Every time during the transient process of shifting, the power will fail and the vehicle and the engine will be unstable. The drastic change of the engine and transmission working condition directly affects the noise change when the gear is shifted.

AMT is the reformed machinery transmission produced currently, which maintains most original components except the shift lever in the manual operation system. It is the successor of the original ones with little cost for improvement, making it rather easy for manufacturers to accept. Its disadvantage lies in its non-dynamic shifting, which has a severe effect on its overall shifting quality although electronic control software can make up for that to some extent.

The stationary shaft- style gear transmission is a traditional device with step shifting, which is widely applied owing to its high efficiency, low cost and sophisticated techniques. It starts with gliding gear transmission, meshed with multi-tooth gear sleeves, and then widely adopts synchronizers to change speed. However, such speech change has difficulty in shifting gears. It will cause the power to fail. Besides, the driver's capability will have an effect on the riding performance. Therefore, people anticipate the automation based on synchronically speeding. The appearance and development of microcomputers provides the possibility of controlling, starting and shifting reasonably.

The engine is the main source of car's noise. This complex power machinery produces various noises which can be divided into gas dynamic noise and surface noise according to the different noise radiation ways. Gas dynamic noise is radiated directly into the air, including induction noise, exhaust noise and fan noise. Surface noise, including combustion noise and mechanical noise, is radiated indirectly outwards through the outside surface of the engine and the vibration of the components connected the engine. The following are several parts resulting in the engine noise.

Combustion noise. Combustion noise is produced by the pressure of the gas with periodical change in the air cylinder when the engine is working, which is decided primarily by the engine's combustion mode and speed. It is not obvious in gasoline engines but weighs more in diesel ones. Whereas, combustion noise mainly refers to noise above $1000 \mathrm{~Hz}$ [15], which needn't pay extra attention to for the traditional noise reduction measures can control it efficiently. 
Mechanical noise. Mechanical noise includes the piston-connecting rod mechanism noise, valve mechanism noise, transmission gear noise and the vibration and noise caused by unbalanced inertia. Those kinds of noise are generally produced by the engine's periodical operation, which is directly related to the engine's structure parameters and dynamic parameters as well as the engine's speed. The vibration and noise generated by unbalanced inertia ought to be paid special attention to.

Induction noise. Induction noise is the main aerodynamic noise just second to exhaust noise, which contains multiple elements. The part with low frequency is periodical pressure pulsation noise.

Exhaust noise. Exhaust noise is the biggest and most important noise source of vehicles' energy, whose fundamental frequency is the engine's igniting frequency and will expand to high harmonics in the whole exhaust noise spectrum.

Fan noise. Although exhaust noise and induction noise are the engine's biggest and most significant sources, their change during the shifting is not evident when it comes to the noise that is studied in this article. The engine's revolving speed changes a lot, leading to a prominent change in the engine's mechanical noise, which is demonstrated on both the noise's sound and frequency distribution. Therefore, the noise change between the shifting is generated directly by the change of the engine's revolving speed. When studying the change, we should emphasize the change of mechanical noise and the change routine of the working condition index.

There are many factors affecting the noise change, since each working condition during the shifting changes a lot. Because the noise fluctuation is mainly caused by the change of the engine's working condition and the gearbox's reaction, the effect that they have on noise during the shifting should be emphasized. The engine's speed fluctuation quantity $(\Delta \mathrm{We})$ to great extent reflects the engine's controlling quality between the shifting. During that time, the engine's speed slows down rapidly in a short time, and then increases gradually, which has a relatively great impact on the engine's combustion and mechanical noises and causes the engine to roar to some degree. The speed fluctuation quantity is inspected to some extent due to the fact that it reflects the engine's own change during the shifting.

The engine's torque change will lead to the fluctuation of the torques of the engine's output shaft and the gearbox's input shaft, thus making the dynamic transmission system change a lot, which affects the sound of the noise. Besides, judging from 3-1, there is an obvious positive correlation between the change of engine's torque and that of the noise, indicating that the change quantity of the engine's torque cannot be overlooked. The inspection reflects the change of the dynamic transmission between the engine and the gearbox.

The AMT clutch is mainly to control the jointing speed that generally goes through three stages: fast, slow, fast, as is shown in 3-11. The second stage affects the noise change directly. If the clutch joints too fast, it will cause shifting impact, thus producing loud noise that is sensitive to human's ears. What's worse, it will even cause the engine to shut down and the vehicle vibrates violently, whose noise is unacceptable. Relatively, if the clutch joints slowly, lower noise will be produced. However, if the clutch joints too slowly, it will take longer time to slide, making its life shorter and the vehicle not stay stable for a long time. It is not beneficial to the stability of noise.

\section{Neural Networks analysis method}

Neural Networks (NN), which is also known as Artificial Neural Network (ANN), is the network where abundant Neurons are linked to one another widely. It is the abstract, simplification and imitation of human's brain, reflecting its essential features. ANN studies human's intelligent 
behavior stemming from the brain's physiological structure to imitate the brain's function of processing information. Neural Networks is the hot research field in recent years, which involves Electronic science and technology, Information and communication engineering, Computer science and technology, Electric engineering, Controlling science and technology, etc. And it is applied to modeling, analysis of time series and mode identification and control .etc.

BP neural network that possesses biological background and accords with the approximation theory is simple and plastic. BP neural network with single implicit strata can approximate an arbitrary nonlinear function, thus being able to gain a better approximate effect. The network trained with high-capacity samples will produce fewer errors when evaluated and better extrapolation ability. Thus, BP neural network is suitable for the analysis and research of shifting noise. Here is the traditional BP neural network structure as Fig .1 shows.
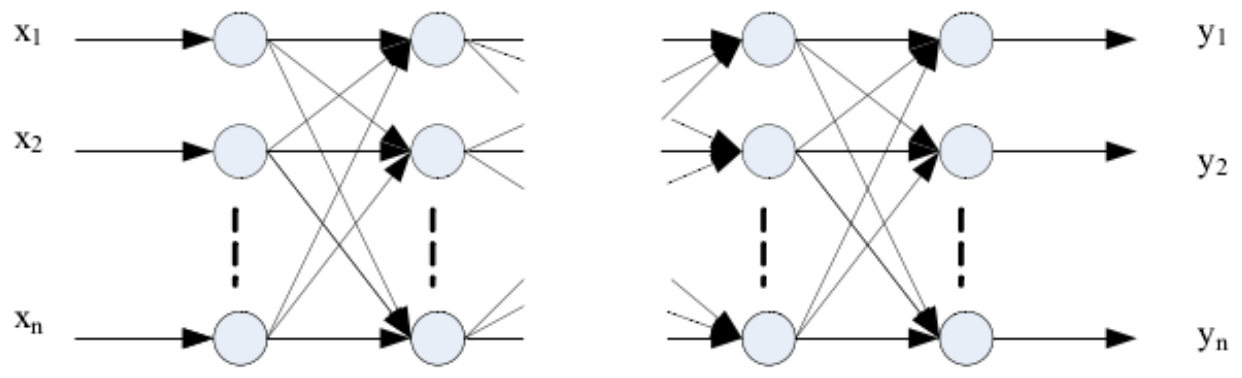

Figure. 1 BP neural network

From figure 1, apart from input and output nodes, there are implicit nodes with one layer or multiple layers. Between the nodes of the same layer exists no coupling. Input signals are transmitted to every implicit node from input nodes by turns and reach the output layer eventually. Nodes output on each layer will only affect those on the next layer.

\section{Conclusion}

In the process of shifting the first gear to the second gear, AMT with its four parameters including the engine's revolving speed quantity $\Delta \mathrm{We}$, torque change quantity $\Delta \mathrm{T}$, clutch jointing speed $\mathrm{VP}$ and shifting time $\mathrm{t}$ has an effect on shifting noise fluctuation quantity $\Delta \mathrm{S}$ P. Choose a proper testing vehicle and start with the first gear to do the experiment of speeding by shifting to higher gears in sequence. Those data should be recorded: the engine's revolving speed, the engine's torque, the clutch's jointing speed, shifting time and noise .etc.

After analyzing the data that are collected, the result shows that $\Delta \mathrm{T}, \mathrm{VP}, \mathrm{t}$ are relatively closer while $\Delta \mathrm{We}$ is different. This comparison experiments can analyze the effect the engine's revolving speed quantity has on shifting noise.

In the two experiments, $\Delta \mathrm{We}$ are $1387 \mathrm{rpm}, 1024 \mathrm{rpm}$ respectively. Because the other three parameters show little difference, it is believed that the difference $(0.29 \mathrm{~Pa})$ of the noise fluctuation between the two experiments is caused mainly by the difference of the engine's revolving speed fluctuation.

In the two experiments, $\Delta \mathrm{T}$ are $29.1 \mathrm{Nm}, 23.6 \mathrm{Nm}$ respectively. Because the other three parameters show little difference, it is believed that the difference $(0.18 \mathrm{~Pa})$ of the noise fluctuation between the two experiments is caused mainly by the difference of the engine's torque fluctuation. 
In the two experiments, $\mathrm{VP}$ are $1.8 \mathrm{~V} / \mathrm{s} 、 2.8 \mathrm{~V} / \mathrm{s}$ respectively while $\mathrm{t}$ are $2.1 \mathrm{~s} 、 1.6 \mathrm{~s}$. Because the other two parameters show little difference, it is believed that the difference $(0.08 \mathrm{~Pa})$ of the noise fluctuation between the two experiments is caused mainly by the difference of the clutch's jointing speed and shifting time.

The comparison experiments indicate that the noise fluctuation of AMT vehicles shifting from the first gear to the second gear is generated primarily by the change of the engine's revolving speed, and then by the change of the engine's torque, while the shifting time and the clutch's jointing speed don't have many effects on it.

\section{Acknowledgements}

This work was financially supported by the key subject building project (vehicle engineering) of Jiangxi University of Technology.

\section{References}

[1] Hornik K, Stinchcombe M, White H. Multilayer feedforward networks are universal approximators[J]. Neural networks, 1989, 2(5): 359-366.

[2] Haykin S, Network N. A comprehensive foundation[J]. Neural Networks, 2004, 2(2004).

[3] Chen J, Zeng Z, Jiang P. Global Mittag-Leffler stability and synchronization of memristor-based fractional-order neural networks[J]. Neural Networks, 2014, 51: 1-8.

[4] Arik S. An improved robust stability result for uncertain neural networks with multiple time delays[J]. Neural Networks, 2014, 54: 1-10.

[5] Cao J, Wan Y. Matrix measure strategies for stability and synchronization of inertial BAM neural network with time delays[J]. Neural Networks, 2014, 53: 165-172.

[6] Dvir E, Strasser G. Does marketing widen borders? Cross-country price dispersion in the European car market[R]. Boston College Department of Economics, 2014.

[7] Vanangamudi S, Prabhakar S, Thamotharan C, et al. Design and Fabrication of Dual Clutch[J]. Middle-East Journal of Scientific Research, 2014, 20(12): 1816-1818.

[8] Cong L, Gao C, Xue Y. Theory Research of Intensive and Automatic Warehouse System Based on Lane-Switch Shuttles[C]//Proceedings of the 5th International Asia Conference on Industrial Engineering and Management Innovation (IEMI2014). Atlantis Press, 2015: 269-274.

[9] Chiatti G, Chiavola O, Recco E. Combustion diagnosis via block vibration signal in common rail diesel engine[J]. International Journal of Engine Research, 2014: 1468087413512311.

[10]Ide T, Otomori M, Leiva J P, et al. Structural optimization methods and techniques to design light and efficient automatic transmission of vehicles with low radiated noise[J]. Structural and Multidisciplinary Optimization, 2014: 1-14. 